\title{
Hybrid method for a class of accretive variational inequalities involving nonexpansive mappings
}

Yaqin Wang ${ }^{1 *}$ and Hong-kun $\mathrm{Xu}^{2}$

\section{"Correspondence:}

wangyaqin0579@126.com

1 Department of Mathematics,

Shaoxing University, Shaoxing,

312000, China

Full list of author information is

available at the end of the article

\begin{abstract}
In this paper we use contractions to regularize a class of accretive variational inequalities and prove the strongly convergence in Banach spaces. We extend the result of Lu et al. (Nonlinear Anal. 71:1032-1041, 2009) to the framework of Banach spaces.

MSC: $47 \mathrm{H} 05 ; 47 \mathrm{H} 09 ; 65 \mathrm{~J} 15$

Keywords: hybrid method; nonexpansive mapping; accretive mapping; variational inequality
\end{abstract}

\section{Introduction}

Let $H$ be a Hilbert space, $C$ be a nonempty closed convex subset of $H$, and $F: C \rightarrow H$ a nonlinear mapping. The set of fixed points of $F$ is denoted by $\operatorname{Fix}(F)$, i.e., $\operatorname{Fix}(F)=\{x \in C$ : $F x=x\}$. A monotone variational inequality problem is to find a point $x^{*}$ with the property

$$
x^{*} \in C \text {, such that }\left\langle F x^{*}, x-x^{*}\right\rangle \geq 0, \quad \forall x \in C,
$$

where $F$ is a monotone operator.

Recently, Lu et al. [1] were concerned with a special class of variational inequalities in which the mapping $F$ is the complement of a nonexpansive mapping and the constraint set is the set of fixed points of another nonexpansive mapping. Namely, they considered the following type of monotone variational inequality (VI) problem:

$$
\text { Find } x^{*} \in \operatorname{Fix}(T) \text {, such that }\left\langle(I-V) x^{*}, x-x^{*}\right\rangle \geq 0, \quad \forall x \in \operatorname{Fix}(T) \text {, }
$$

where $T, V: C \rightarrow C$ are nonexpansive mappings and $\operatorname{Fix}(T) \neq \emptyset$.

Hybrid methods for solving VI (1) were studied by Yamada [2], where $F$ is Lipschitzian and strongly monotone. However, his methods do not apply to the variational inequality (2) since the mapping $I-V$ fails, in general, to be strongly monotone, though it is Lipschitzian. Therefore, other hybrid methods have to be sought. Recently, Moudafi and Mainge [3] studied VI (2) by regularizing the mapping $t S+(1-t) T$ and defined $\left\{x_{s, t}\right\}$ as the unique fixed point of the equation

$$
x_{s, t}=s f\left(x_{s, t}\right)+(1-s)\left[t S\left(x_{s, t}\right)+(1-t) T\left(x_{s, t}\right)\right], \quad s, t \in(0,1) .
$$

O2014 Wang and Xu; licensee Springer. This is an Open Access article distributed under the terms of the Creative Commons Attribution License (http://creativecommons.org/licenses/by/2.0), which permits unrestricted use, distribution, and reproduction in any medium, provided the original work is properly cited. 
Since Moudafi and Mainge's regularization depends on $t$, the convergence of the scheme (3) is more complicated. Very recently, Lu et al. [1] studied VI (2) by regularizing the mapping $S$ and defined $\left\{x_{s, t}\right\}$ as the unique fixed point of the equation

$$
x_{s, t}=s\left[t f\left(x_{s, t}\right)+(1-t) S\left(x_{s, t}\right)\right]+(1-s) T\left(x_{s, t}\right), \quad s, t \in(0,1) .
$$

Note that Lu et al.'s regularization (4) no longer depends on $t$.

Motivated and inspired by the result of Lu et al. [1], we put forward a question: Can this implicit hybrid method [1] in Hilbert spaces be extended to the framework of Banach spaces? In this paper, we give a positive answer.

Throughout this paper, we always assume that $E$ is a real Banach space. Let $C$ be a nonempty closed convex subset of $E$. Let $F: C \rightarrow E$ be a nonlinear mapping.

In this paper, we consider the following type of accretive variational inequality problem:

$$
x^{*} \in \operatorname{Fix}(T) \text {, such that }\left\langle(I-S) x^{*}, j\left(x-x^{*}\right)\right\rangle \geq 0, \quad \forall x \in \operatorname{Fix}(T),
$$

where $S, T: C \rightarrow C$ are two nonexpansive mappings with the set of fixed point $\operatorname{Fix}(T) \neq \emptyset$. Let $\Omega$ denote the set of solutions of VI (5) and assume that $\Omega$ is nonempty.

\section{Preliminaries}

Let $E$ be a real Banach space and $J$ be the normalized duality mapping from $E$ into $2^{E^{*}}$ given by

$$
J(x)=\left\{x^{*} \in E^{*}:\left\langle x, x^{*}\right\rangle=\|x\|\left\|x^{*}\right\|,\|x\|=\left\|x^{*}\right\|\right\}
$$

for all $x \in E$, where $E^{*}$ denotes the dual space of $E$ and $\langle\cdot, \cdot\rangle$ the generalized duality pairing between $E$ and $E^{*}$.

Let $C$ be a nonempty closed convex subset of a real Banach space $E$. Recall the following concepts of mappings.

(i) A mapping $f: C \rightarrow C$ is a $\rho$-contraction if $\rho \in[0,1)$ and the following property is satisfied:

$$
\|f(x)-f(y)\| \leq \rho\|x-y\|, \quad \forall x, y \in C .
$$

(ii) A mapping $T: C \rightarrow C$ is nonexpansive provided

$$
\|T x-T y\| \leq\|x-y\|, \quad \forall x, y \in C .
$$

(iii) A mapping $F: C \rightarrow E$ is

(a) accretive if for any $x, y \in C$ there exists $j(x-y) \in J(x-y)$ such that

$$
\langle F x-F y, j(x-y)\rangle \geq 0
$$

(b) strictly accretive if $F$ is accretive and the equality in (a) holds if and only if $x=y$;

(c) $\beta$-strongly accretive if for any $x, y \in C$ there exists $j(x-y) \in J(x-y)$ such that

$$
\langle F x-F y, j(x-y)\rangle \geq \beta\|x-y\|^{2}
$$

for some real constant $\beta>0$. 
Let $\varphi:[0, \infty):=R^{+} \rightarrow R^{+}$be a continuous strictly increasing function such that $\varphi(0)=0$ and $\varphi(t) \rightarrow \infty$ as $t \rightarrow \infty$. This function $\varphi$ is called a gauge function. The duality mapping $J_{\varphi}: E \rightarrow E^{*}$ associated with a gauge function $\varphi$ is defined by

$$
J_{\varphi}(x)=\left\{x^{*} \in E^{*}:\left\langle x, x^{*}\right\rangle=\|x\| \varphi(\|x\|),\left\|x^{*}\right\|=\varphi(\|x\|)\right\}, \quad \forall x \in E .
$$

In the case that $\varphi(t)=t, J_{\varphi}=J$, where $J$ is the normalized duality mapping. Clearly, the relation $J_{\varphi}(x)=\frac{\varphi(\|x\|)}{\|x\|} J(x), \forall x \neq 0$ holds (see [4]).

Following Browder [4], we say that a Banach space $E$ has a weakly continuous duality mapping if there exists a gauge $\varphi$ for which the duality mapping $J_{\varphi}(x)$ is single valued and weak-to-weak* sequentially continuous (i.e., if $\left\{x_{n}\right\}$ is a sequence in $E$ weakly convergent to a point $x$, then the sequence $J_{\varphi}\left(x_{n}\right)$ converges weakly* to $\left.J_{\varphi}(x)\right)$. It is well known that $l^{p}$ has a weakly continuous duality mapping with a gauge function $\varphi(t)=t^{p-1}$ for all $1<p<\infty$. Set

$$
\Phi(t)=\int_{0}^{t} \varphi(\tau) d \tau, \quad t>0
$$

then

$$
J_{\varphi}(x)=\partial \Phi(\|x\|), \quad \forall x \in E,
$$

where $\partial$ denotes the sub-differential in the sense of convex analysis.

Remark 2.1 If $J_{\varphi}$ is weak-to-weak* sequentially continuous, then $J$ is strong-to-weak* sequentially continuous.

Indeed, if $x_{n} \rightarrow x$ strongly, then $x_{n} \rightarrow x$ weakly, $J_{\varphi}\left(x_{n}\right)$ converges weakly* to $J_{\varphi}(x)$ and $\varphi\left(\left\|x_{n}\right\|\right) \rightarrow \varphi(\|x\|)$ strongly. Since $J_{\varphi}(x) \frac{\|x\|}{\varphi(\|x\|)}=J(x), \forall x \neq 0$, for any $y \in E$, we have

$$
\begin{aligned}
&\left|\left\langle y, J\left(x_{n}\right)\right\rangle-\langle y, J(x)\rangle\right| \\
&=\left|\left\langle y, J_{\varphi}\left(x_{n}\right) \frac{\left\|x_{n}\right\|}{\varphi\left(\left\|x_{n}\right\|\right)}\right\rangle-\left\langle y, J_{\varphi}(x) \frac{\|x\|}{\varphi(\|x\|)}\right\rangle\right| \\
& \leq\left|\left\langle y, J_{\varphi}\left(x_{n}\right) \frac{\left\|x_{n}\right\|}{\varphi\left(\left\|x_{n}\right\|\right)}\right\rangle-\left\langle y, J_{\varphi}\left(x_{n}\right) \frac{\|x\|}{\varphi(\|x\|)}\right\rangle\right|+\left|\left\langle y, J_{\varphi}\left(x_{n}\right) \frac{\|x\|}{\varphi(\|x\|)}\right\rangle-\left\langle y, J_{\varphi}(x) \frac{\|x\|}{\varphi(\|x\|)}\right\rangle\right| \\
& \leq\|y\| \varphi\left(\left\|x_{n}\right\|\right) \frac{\left|\left\|x_{n}\right\| \varphi(\|x\|)-\|x\| \varphi\left(\left\|x_{n}\right\|\right)\right|}{\varphi\left(\left\|x_{n}\right\|\right) \varphi(\|x\|)}+\frac{\|x\|}{\varphi(\|x\|)}\left|\left\langle y, J_{\varphi}\left(x_{n}\right)\right\rangle-\left\langle y, J_{\varphi}(x)\right\rangle\right| \\
& \leq\|y\| \frac{\left\|x_{n}\right\|\left|\varphi(\|x\|)-\varphi\left(\left\|x_{n}\right\|\right)\right|+\left|\left\|x_{n}\right\|-\|x\|\right| \varphi\left(\left\|x_{n}\right\|\right)}{\varphi(\|x\|)} \\
&+\frac{\|x\|}{\varphi(\|x\|)} \mid\left\langle y, J_{\varphi}\left(x_{n}\right)\right\rangle-\left\langle y, J_{\varphi}(x)\right\rangle .
\end{aligned}
$$

Letting $n \rightarrow \infty$, we have

$$
\lim _{n \rightarrow \infty}\left\langle y, J\left(x_{n}\right)\right\rangle=\langle y, J(x)\rangle,
$$

i.e., $J$ is strong-to-weak* sequentially continuous. 
Lemma 2.1 ([5, Lemma 2.1]) Assume that a Banach space E has a weakly continuous duality mapping $J_{\varphi}$ with a gauge $\varphi$. For all $x, y \in E$, the following inequality holds:

$$
\Phi(\|x+y\|) \leq \Phi(\|x\|)+\left\langle y, J_{\varphi}(x+y)\right\rangle .
$$

In particular, for all $x, y \in E$,

$$
\|x+y\|^{2} \leq\|x\|^{2}+2\langle y, J(x+y)\rangle .
$$

Lemma 2.2 (see [6]) Let $C$ be a nonempty closed convex subset of a real Banach space $E$. Assume that $F: C \rightarrow E$ is accretive and weakly continuous along segments; that is $F(x+$ $t y) \rightarrow F(x)$ as $t \rightarrow 0$. Then the variational inequality

$$
x^{*} \in C, \quad\left\langle F x^{*}, j\left(x-x^{*}\right)\right| \geq 0, \quad \forall x \in C
$$

is equivalent to the dual variational inequality

$$
x^{*} \in C, \quad\left\langle F x, j\left(x-x^{*}\right)\right\rangle \geq 0, \quad \forall x \in C .
$$

\section{Main results}

In this section, we introduce an implicit algorithm and prove this algorithm converges strongly to $x^{*}$ which solves VI (5). Let $C$ be a nonempty closed convex subset of a real Banach space $E$. Let $f: C \rightarrow C$ be a contraction and $S, T: C \rightarrow C$ be two nonexpansive mappings. For $s, t \in(0,1)$, we define the following mapping:

$$
x \mapsto W_{s, t} x:=s[t f(x)+(1-t) S x]+(1-s) T x .
$$

It is obvious that $W_{s, t}: C \rightarrow C$ is a contraction. So the contraction $W_{s, t}$ has a unique fixed point which is denoted $x_{s, t}$. Namely,

$$
x_{s, t}=s\left[t f\left(x_{s, t}\right)+(1-t) S\left(x_{s, t}\right)\right]+(1-s) T\left(x_{s, t}\right), \quad s, t \in(0,1) .
$$

Theorem 3.1 Let $C$ be a nonempty closed convex subset of a reflexive Banach space $E$ which has a weakly continuous duality map $J_{\varphi}(x)$ with the gauge $\varphi$. Let $f: C \rightarrow C$ be a contraction with constant $\rho>0$ and $S, T: C \rightarrow C$ be two nonexpansive mappings with $\operatorname{Fix}(T) \neq \emptyset$. Suppose that the solution set $\Omega$ of VI (5) is nonempty. Let, for each $(s, t) \in(0,1)^{2}$, $\left\{x_{s, t}\right\}$ be defined implicitly by (6). Then, for each fixed $t \in(0,1)$, the net $\left\{x_{s, t}\right\}$ converges in norm, as $s \rightarrow 0$, to a point $x_{t} \in \mathrm{Fix}(T)$. Moreover, as $t \rightarrow 0$, the net $\left\{x_{t}\right\}$ converges in norm to the unique solution $x^{*}$ of the following variational inequality:

$$
x^{*} \in \Omega, \quad\left\langle(I-f) x^{*}, J\left(x-x^{*}\right)\right\rangle \geq 0, \quad \forall x \in \Omega .
$$

Hence, for each null sequence $\left\{t_{n}\right\}$ in $(0,1)$, there exists another null sequence $\left\{s_{n}\right\}$ in $(0,1)$, such that the sequence $x_{s_{n}, t_{n}} \rightarrow x^{*}$ in norm as $n \rightarrow \infty$.

Proof Step 1. For each fixed $t \in(0,1)$, the net $\left\{x_{s, t}\right\}$ is bounded. 
For any $z \in \operatorname{Fix}(T)$, we have

$$
\begin{aligned}
& \left\|s\left[t\left(f\left(x_{s, t}\right)-f(z)\right)+(1-t)\left(S\left(x_{s, t}\right)-S(z)\right)\right]+(1-s)\left(T\left(x_{s, t}\right)-z\right)\right\| \\
& \quad \leq s\left\|t\left(f\left(x_{s, t}\right)-f(z)\right)+(1-t)\left(S\left(x_{s, t}\right)-S(z)\right)\right\|+(1-s)\left\|T\left(x_{s, t}\right)-T z\right\| \\
& \quad \leq s t\left\|f\left(x_{s, t}\right)-f(z)\right\|+(1-t) s\left\|S\left(x_{s, t}\right)-S(z)\right\|+(1-s)\left\|x_{s, t}-z\right\| \\
& \quad \leq s t \rho\left\|x_{s, t}-z\right\|+(1-t) s\left\|x_{s, t}-z\right\|+(1-s)\left\|x_{s, t}-z\right\| \\
& \quad=(1-s t(1-\rho))\left\|x_{s, t}-z\right\| .
\end{aligned}
$$

Combining the above inequality and Lemma 2.1, we obtain

$$
\begin{aligned}
\Phi\left(\left\|x_{s, t}-z\right\|\right)= & \Phi\left(\| s\left[t\left(f\left(x_{s, t}\right)-f(z)\right)+(1-t)\left(S\left(x_{s, t}\right)-S(z)\right)\right]\right. \\
& \left.+(1-s)\left(T\left(x_{s, t}\right)-z\right)+s t(f(z)-z)+s(1-t)(S(z)-z) \|\right) \\
\leq & \Phi\left(\left\|s\left[t\left(f\left(x_{s, t}\right)-f(z)\right)+(1-t)\left(S\left(x_{s, t}\right)-S(z)\right)\right]+(1-s)\left(T\left(x_{s, t}\right)-z\right)\right\|\right) \\
& +s t\left\langle f(z)-z, J_{\varphi}\left(x_{s, t}-z\right)\right\rangle+(1-t) s\left\langle S(z)-z, J_{\varphi}\left(x_{s, t}-z\right)\right\rangle \\
\leq & \Phi\left((1-s t(1-\rho))\left\|x_{s, t}-z\right\|\right)+s t\left|f(z)-z, J_{\varphi}\left(x_{s, t}-z\right)\right\rangle \\
& +(1-t) s\left\langle S(z)-z, J_{\varphi}\left(x_{s, t}-z\right)\right\rangle \\
\leq & (1-s t(1-\rho)) \Phi\left(\left\|x_{s, t}-z\right\|\right)+s t\left|f(z)-z, J_{\varphi}\left(x_{s, t}-z\right)\right\rangle \\
& +(1-t) s\left(S(z)-z, J_{\varphi}\left(x_{s, t}-z\right)\right\rangle,
\end{aligned}
$$

which implies that

$$
\Phi\left(\left\|x_{s, t}-z\right\|\right) \leq \frac{t}{t(1-\rho)}\left\langle f(z)-z, J_{\varphi}\left(x_{s, t}-z\right)\right\rangle+\frac{1-t}{t(1-\rho)}\left\langle S(z)-z, J_{\varphi}\left(x_{s, t}-z\right)\right\rangle .
$$

Taking $\varphi(t)=t$, then $J_{\varphi}=J$ and $\Phi(t)=\frac{t^{2}}{2}$, from (8) we have

$$
\begin{aligned}
\left\|x_{s, t}-z\right\|^{2} & \leq \frac{2 t}{t(1-\rho)}\left\langle f(z)-z, J\left(x_{s, t}-z\right)\right\rangle+\frac{2(1-t)}{t(1-\rho)}\left\langle S(z)-z, J\left(x_{s, t}-z\right)\right\rangle \\
& \leq \frac{2 t}{t(1-\rho)}\|f(z)-z\|\left\|x_{s, t}-z\right\|+\frac{2(1-t)}{t(1-\rho)}\|S(z)-z\|\left\|x_{s, t}-z\right\|,
\end{aligned}
$$

which implies that

$$
\left\|x_{s, t}-z\right\| \leq \frac{2}{t(1-\rho)} \max \{\|f(z)-z\|,\|S(z)-z\|\} .
$$

So for each fixed $t \in(0,1),\left\{x_{s, t}\right\}$ is bounded, furthermore $\left\{f\left(x_{s, t}\right)\right\},\left\{S\left(x_{s, t}\right)\right\}$ and $\left\{T\left(x_{s, t}\right)\right\}$ are all bounded.

Step 2. $x_{s, t} \rightarrow x_{t} \in \operatorname{Fix}(T)$ as $s \rightarrow 0$.

From (6) and the boundedness of the sequences $\left\{f\left(x_{s, t}\right)\right\},\left\{S\left(x_{s, t}\right)\right\}$ and $\left\{T\left(x_{s, t}\right)\right\}$, for each fixed $t \in(0,1)$ we have

$$
\left\|x_{s, t}-T x_{s, t}\right\|=s\left\|t f\left(x_{s, t}\right)+(1-t) S\left(x_{s, t}\right)-T x_{s, t}\right\| \rightarrow 0 \quad(s \rightarrow 0) .
$$


Assume that $\left\{s_{n}\right\} \subset(0,1)$ is such that $s_{n} \rightarrow 0(n \rightarrow \infty)$. From (8), for any $z \in \operatorname{Fix}(T)$, we have

$$
\Phi\left(\left\|x_{s_{n}, t}-z\right\|\right) \leq \frac{t}{t(1-\rho)}\left\langle f(z)-z, J_{\varphi}\left(x_{s_{n}, t}-z\right)\right\rangle+\frac{1-t}{t(1-\rho)}\left\langle S(z)-z, J_{\varphi}\left(x_{s_{n}, t}-z\right)\right\rangle .
$$

Since $\left\{x_{s_{n}, t}\right\}$ is bounded, without loss of generality, we may assume that $\left\{x_{s_{n}, t}\right\}$ converges weakly to a point $x_{t}$ as $n \rightarrow \infty$. This together with (10) implies that $x_{t} \in \operatorname{Fix}(T)$. Taking $z=x_{t}$ in (11), we have

$$
\Phi\left(\left\|x_{s_{n}, t}-x_{t}\right\|\right) \leq \frac{1}{t(1-\rho)}\left\langle t f\left(x_{t}\right)+(1-t) S\left(x_{t}\right)-x_{t}, J_{\varphi}\left(x_{s_{n}, t}-x_{t}\right)\right\rangle .
$$

Since $J_{\varphi}$ is weakly continuous, it follows from (12) that $\Phi\left(\left\|x_{s_{n}, t}-x_{t}\right\|\right) \rightarrow 0$ as $n \rightarrow \infty$, which implies that $x_{s_{n}, t} \rightarrow x_{t}$ strongly. This has proved the relative norm compactness of the net $\left\{x_{s, t}\right\}$ as $s \rightarrow 0$.

Taking $s=s_{n}$ in (9), we have

$$
\left\|x_{s_{n}, t}-z\right\|^{2} \leq \frac{2 t}{t(1-\rho)}\left\langle f(z)-z, J\left(x_{s_{n}, t}-z\right)\right\rangle+\frac{2(1-t)}{t(1-\rho)}\left\langle S(z)-z, J\left(x_{s_{n}, t}-z\right)\right\rangle .
$$

Since $J_{\varphi}$ is weakly continuous, then by Remark $2.1, J$ is strong-to-weak* sequentially continuous. Let $s_{n} \rightarrow 0$ in the above inequality, we have

$$
\left\|x_{t}-z\right\|^{2} \leq \frac{2 t}{t(1-\rho)}\left\langle f(z)-z, J\left(x_{t}-z\right)\right\rangle+\frac{2(1-t)}{t(1-\rho)}\left\langle S(z)-z, J\left(x_{t}-z\right)\right\rangle .
$$

Hence we obtain

$$
x_{t} \in \operatorname{Fix}(T), \quad\left\langle t f(z)+(1-t) S(z)-z, J\left(x_{t}-z\right)\right\rangle \geq 0, \quad \forall z \in \operatorname{Fix}(T) .
$$

This together with Lemma 2.2, we have

$$
x_{t} \in \operatorname{Fix}(T), \quad\left\langle t f\left(x_{t}\right)+(1-t) S\left(x_{t}\right)-x_{t}, J\left(x_{t}-z\right)\right\rangle \geq 0, \quad \forall z \in \operatorname{Fix}(T) .
$$

Next, we prove that the entire net $\left\{x_{s, t}\right\}$ converges strongly to $x_{t}$ as $s \rightarrow 0$. We assume that $x_{s_{n}^{\prime}, t} \rightarrow x_{t}^{\prime}$ where $s_{n}^{\prime} \rightarrow 0$. Similar to the above proof, we have $x_{t}^{\prime} \in \operatorname{Fix}(T)$ and

$$
x_{t}^{\prime} \in \operatorname{Fix}(T), \quad\left\langle t f\left(x_{t}^{\prime}\right)+(1-t) S\left(x_{t}^{\prime}\right)-x_{t}^{\prime}, J\left(x_{t}^{\prime}-z\right)\right\rangle \geq 0, \quad \forall z \in \operatorname{Fix}(T) .
$$

Taking $z=x^{\prime}(t)$ and $z=x_{t}$ in (13) and (14), respectively, we have

$$
\begin{aligned}
& t\left\{f\left(x_{t}\right)-x_{t}, J\left(x_{t}-x_{t}^{\prime}\right)\right\rangle+(1-t)\left\langle S\left(x_{t}\right)-x_{t}, J\left(x_{t}-x_{t}^{\prime}\right)\right\rangle \geq 0, \\
& t\left\{f\left(x_{t}^{\prime}\right)-x_{t}^{\prime}, J\left(x_{t}^{\prime}-x_{t}\right)\right\rangle+(1-t)\left\langle S\left(x_{t}^{\prime}\right)-x_{t}^{\prime}, J\left(x_{t}^{\prime}-x_{t}\right)\right\rangle \geq 0 .
\end{aligned}
$$

Adding up the above two inequalities yields

$$
t\left\langle(I-f) x_{t}-(I-f) x_{t}^{\prime}, J\left(x_{t}-x_{t}^{\prime}\right)\right\rangle+(1-t)\left\langle(I-S) x_{t}-(I-S) x_{t}^{\prime}, J\left(x_{t}-x_{t}^{\prime}\right)\right\rangle \leq 0 .
$$


Since

$$
\begin{aligned}
& \left\langle(I-f) x_{t}-(I-f) x_{t}^{\prime}, J\left(x_{t}-x_{t}^{\prime}\right)\right\rangle \geq(1-\rho)\left\|x_{t}-x_{t}^{\prime}\right\|^{2}, \\
& \left\langle(I-S) x_{t}-(I-S) x_{t}^{\prime}, J\left(x_{t}-x_{t}^{\prime}\right)\right\rangle \geq 0,
\end{aligned}
$$

we obtain

$$
(1-\rho)\left\|x_{t}-x_{t}^{\prime}\right\|^{2} \leq 0
$$

i.e., $x_{t}=x_{t}^{\prime}$. So the entire net $\left\{x_{s, t}\right\}$ converges in norm to $x_{t} \in \operatorname{Fix}(T)$ as $s \rightarrow 0$.

Step 3. The net $\left\{x_{t}\right\}$ is bounded.

For any $y \in \Omega$, taking $z=y$ in (13), we have

$$
\left\langle t f\left(x_{t}\right)+(1-t) S\left(x_{t}\right)-x_{t}, J\left(x_{t}-y\right)\right\rangle \geq 0
$$

which together with the fact of $y \in \Omega$ implies that

$$
\begin{aligned}
& t\left\langle(I-f) x_{t}-(I-f) y, J\left(x_{t}-y\right)\right\rangle+(1-t)\left\langle(I-S) x_{t}-(I-S) y, J\left(x_{t}-y\right)\right\rangle \\
& \quad \leq t\left\langle f(y)-y, J\left(x_{t}-y\right)\right\rangle+(1-t)\left\langle S y-y, J\left(x_{t}-y\right)\right\rangle \\
& \quad \leq t\left\langle f(y)-y, J\left(x_{t}-y\right)\right\rangle .
\end{aligned}
$$

Since $I-f$ is strongly accretive and $I-S$ is accretive, we obtain

$$
\begin{aligned}
& \left\langle(I-f) x_{t}-(I-f) y, J\left(x_{t}-y\right)\right\rangle \geq(1-\rho)\left\|x_{t}-y\right\|^{2}, \\
& \left\langle(I-S) x_{t}-(I-S) y, J\left(x_{t}-y\right)\right\rangle \geq 0 .
\end{aligned}
$$

It follows from (15)-(17) that

$$
\begin{aligned}
\left\|x_{t}-y\right\|^{2} & \leq \frac{1}{1-\rho}\left\langle f(y)-y, J\left(x_{t}-y\right)\right\rangle \\
& \leq \frac{1}{1-\rho}\|f(y)-y\|\left\|x_{t}-y\right\| .
\end{aligned}
$$

Hence we have

$$
\left\|x_{t}-y\right\| \leq \frac{1}{1-\rho}\|f(y)-y\|, \quad \forall t \in(0,1)
$$

Step 4. The net $x_{t} \rightarrow x^{*} \in \Omega$ which solves VI (7).

First, the uniqueness of the solution of VI (7) is obvious. We denote the unique solution by $x^{*}$.

Next we prove that $\omega_{w}\left(x_{t}\right) \subset \Omega$, i.e., if $\left\{t_{n}\right\}$ is a null sequence in $(0,1)$ such that $x_{t_{n}} \rightarrow x^{\prime}$ weakly as $n \rightarrow \infty$, then $x^{\prime} \in \Omega$. Indeed, since $\left\{x_{t}\right\} \subset \operatorname{Fix}(T)$, then $x^{\prime} \in \operatorname{Fix}(T)$. Since $I-S$ is accretive, for any $z \in \operatorname{Fix}(T)$ we have

$$
\left\langle(I-S) z, J\left(z-x_{t}\right)\right\rangle \geq\left\langle(I-S) x_{t}, J\left(z-x_{t}\right)\right\rangle .
$$


It follows from (13) that

$$
\left\langle(I-S) x_{t}, J\left(z-x_{t}\right)\right\rangle \geq \frac{t}{1-t}\left\langle(I-f) x_{t}, J\left(x_{t}-z\right)\right\rangle .
$$

By virtue of (19) and (20), we have

$$
\left\langle(I-S) z, J\left(z-x_{t}\right)\right\rangle \geq \frac{t}{1-t}\left\langle(I-f) x_{t}, J\left(x_{t}-z\right)\right\rangle
$$

furthermore, we get

$$
\begin{aligned}
\left\langle(I-S) z, J_{\varphi}\left(z-x_{t}\right)\right\rangle & \geq \frac{t}{(1-t)}\left\langle(I-f) x_{t}, J_{\varphi}\left(x_{t}-z\right)\right\rangle, \\
& \geq-\frac{t}{(1-t)}\left(\left\|x_{t}\right\|+\left\|f\left(x_{t}\right)\right\|\right) \varphi\left(\left\|x_{t}-z\right\|\right) .
\end{aligned}
$$

Letting $t=t_{n} \rightarrow 0(n \rightarrow \infty)$ in the above inequality, since $\left\{x_{t}\right\}$ is bounded and $\varphi$ is a continuous strictly increasing function, we have

$$
\left\langle(I-S) z, J_{\varphi}\left(z-x^{\prime}\right)\right\rangle \geq 0, \quad \forall z \in \operatorname{Fix}(T) .
$$

This implies that

$$
\left\langle(I-S) z, J\left(z-x^{\prime}\right)\right\rangle \geq 0, \quad \forall z \in \operatorname{Fix}(T)
$$

hence from the above inequality and Lemma 2.2, we have

$$
\left\langle(I-S) x^{\prime}, J\left(z-x^{\prime}\right)\right\rangle \geq 0, \quad \forall z \in \operatorname{Fix}(T)
$$

i.e., $x^{\prime} \in \Omega$.

Next we show that $x^{\prime}$ is the solution of VI (7). Taking $y=x^{\prime}$ and $t=t_{n}$ in (18), we obtain

$$
\begin{aligned}
\left\|x_{t_{n}}-x^{\prime}\right\|^{2} & \leq \frac{1}{1-\rho}\left\langle f\left(x^{\prime}\right)-x^{\prime}, J\left(x_{t_{n}}-x^{\prime}\right)\right\rangle \\
& =\frac{1}{1-\rho}\left\langle f\left(x^{\prime}\right)-x^{\prime}, J_{\varphi}\left(x_{t_{n}}-x^{\prime}\right)\right\rangle \frac{\left\|x_{t_{n}}-x^{\prime}\right\|}{\varphi\left(\left\|x_{t_{n}}-x^{\prime}\right\|\right)},
\end{aligned}
$$

which implies that

$$
\left\|x_{t_{n}}-x^{\prime}\right\| \varphi\left(\left\|x_{t_{n}}-x^{\prime}\right\|\right) \leq \frac{1}{1-\rho}\left\langle f\left(x^{\prime}\right)-x^{\prime}, J_{\varphi}\left(x_{t_{n}}-x^{\prime}\right)\right\rangle .
$$

Since $x_{t_{n}} \rightarrow x^{\prime}$ weakly and $J_{\varphi}$ is weakly continuous, let $t_{n} \rightarrow 0$ in (21), we get

$$
\left\|x_{t_{n}}-x^{\prime}\right\| \varphi\left(\left\|x_{t_{n}}-x^{\prime}\right\|\right) \rightarrow 0 \quad(n \rightarrow \infty)
$$

which together with the property of $\varphi$ implies that $x_{t_{n}} \rightarrow x^{\prime}$ in norm. It follows from (15) and (17) that

$$
\left\langle(I-f) x_{t}, J\left(x_{t}-y\right)\right\rangle \leq 0 .
$$


Since $J$ is strong-to-weak* sequentially continuous and $f$ is a contraction, we have

$$
\begin{aligned}
&\left|\left\langle(I-f) x_{t_{n}}, J\left(x_{t_{n}}-y\right)\right\rangle-\left\langle(I-f) x^{\prime}, J\left(x^{\prime}-y\right)\right\rangle\right| \\
& \leq\left|\left\langle(I-f) x_{t_{n}}, J\left(x_{t_{n}}-y\right)\right\rangle-\left\langle(I-f) x^{\prime}, J\left(x_{t_{n}}-y\right)\right\rangle\right| \\
& \quad+\left|\left\langle(I-f) x^{\prime}, J\left(x_{t_{n}}-y\right)\right\rangle-\left\langle(I-f) x^{\prime}, J\left(x^{\prime}-y\right)\right\rangle\right| \\
& \leq\left|\left\langle(I-f) x_{t_{n}}, J\left(x_{t_{n}}-y\right)\right\rangle-\left\langle(I-f) x^{\prime}, J\left(x_{t_{n}}-y\right)\right\rangle\right| \\
& \quad+\left|\left\langle(I-f) x^{\prime}, J\left(x_{t_{n}}-y\right)\right\rangle-\left\langle(I-f) x^{\prime}, J\left(x^{\prime}-y\right)\right\rangle\right| \\
& \leq(1+\rho)\left\|x_{t_{n}}-x^{\prime}\right\|\left\|x_{t_{n}}-y\right\| \\
&+\left|\left\langle(I-f) x^{\prime}, J\left(x_{t_{n}}-y\right)\right\rangle-\left\langle(I-f) x^{\prime}, J\left(x^{\prime}-y\right)\right\rangle\right| \rightarrow 0 \quad(n \rightarrow \infty) .
\end{aligned}
$$

Letting $t=t_{n} \rightarrow 0(n \rightarrow \infty)$ in (22) and combining (23) we have

$$
\left\langle(I-f) x^{\prime}, J\left(x^{\prime}-y\right)\right\rangle \leq 0, \quad \forall y \in \Omega .
$$

So $x^{\prime}$ is the solution of VI (7). By uniqueness, we have $x^{\prime}=x^{*}$. Therefore, $x_{t} \rightarrow x^{*}$ in norm as $t \rightarrow 0$. The proof is complete.

\section{Competing interests}

The authors declare that they have no competing interests.

\section{Authors' contributions}

All authors contributed equally to the writing of this paper. All authors read and approved the final manuscript.

\section{Author details}

1Department of Mathematics, Shaoxing University, Shaoxing, 312000, China. ${ }^{2}$ Department of Applied Mathematics,

National Sun Yat-sen University, Kaohsiung, 80424, Taiwan.

\section{Acknowledgements}

The first author was supported by Zhejiang Provincial Natural Science Foundation of China under Grant (no. LQ13A010007, no. LY14A010006) and the China Postdoctoral Science Foundation Funded Project (no. 2012M511928).

Received: 21 March 2014 Accepted: 22 May 2014 Published: 29 May 2014

\section{References}

1. Lu, XW, Xu, HK, Yin, XM: Hybrid methods for a class of monotone variational inequalities. Nonlinear Anal. 71, 1032-1041 (2009)

2. Yamada, I: The hybrid steepest descent for the variational inequality problems over the intersection of fixed point sets of nonexpansive mappings. In: Inherently Parallel Algorithms in Feasibility and Optimization and Their Applications, pp. 473-504. Elsevier, New York (2001)

3. Moudafi, A, Mainge, P-E: Towards viscosity approximations of hierarchical fixed-points problems. Fixed Point Theory Appl. 2006, Article ID 95453 (2006)

4. Browder, FE: Convergence theorems for sequences of nonlinear operators in Banach spaces. Math. Z. 100, 201-225 (1967)

5. Xu, HK: A strong convergence theorem for contraction semigroup in Banach spaces. Bull. Aust. Math. Soc. 72, 371-379 (2005)

6. Wang, YQ, Chen, RD: Hybrid methods for accretive variational inequalities involving pseudocontractions in Banach spaces. Fixed Point Theory Appl. 2011, 63 (2011)

10.1186/1029-242X-2014-217

Cite this article as: Wang and $\mathrm{Xu}$ : Hybrid method for a class of accretive variational inequalities involving nonexpansive mappings. Journal of Inequalities and Applications 2014, 2014:217 